K. Watanabe

Nagoya Math. J.

Vol. 77 (1980), 89-98

\title{
INVARIANT SUBRINGS WHICH ARE COMPLETE INTERSECTIONS, I \\ (INVARIANT SUBRINGS OF FINITE ABELIAN GROUPS)
}

\author{
KEIICHI WATANABE*
}

\section{Introduction}

Let $G$ be a finite subgroup of $G L(n, C)(C$ is the field of complex numbers). Then $G$ acts naturally on the polynomial ring $S=C\left[X_{1}, \cdots, X_{n}\right]$. We consider the following

Problem. When is the invariant subring $S^{a}$ a complete intersection?

In this paper, we treat the case where $G$ is a finite Abelian group. We can solve the problem completely. The result is stated in Theorem 2.1 .

\section{Construction of the groups and the invariant subrings}

First, let us fix some notations.

$Z$ is the ring of integers.

$N$ is the additive semigroup of nonnegative integers.

$Z_{+}$is the set of positive integers.

$C$ is the field of complex numbers.

$S=C\left[X_{1}, \cdots, X_{n}\right]$.

$G$ is a finite Abelian subgroup of $G L(n, C)$. It is well known that $G$ is diagonalizable. So we will always assume that every element of $G$ is a diagonal matrix.

$e_{m}$ is a primitive $m$-th root of unity.

$I=\{1, \cdots, n\}$ (the index set of variables).

$(a ; i)$ (resp. $(a, b ; i, j))$ is the diagonal matrix whose $(i, i)$ component is $a$ (resp, $(i, i)$ component is $a$ and $(j, j)$ component is $b$ ) and the other

* The author was partially supported by Matsunaga Science Foundation.

Received December 12, 1978. 
diagonal components are 1. For example, if $n=3$,

$$
(a ; 2)=\left[\begin{array}{lll}
1 & & \\
& a & \\
& & 1
\end{array}\right] \text { and }(a, b ; 1,3)=\left[\begin{array}{lll}
a & & \\
& 1 & \\
& & b
\end{array}\right] \text {. }
$$

Definition 1.1. A special datum $D$ is a couple $(D, w)$ where $D$ is a set of subsets of $I$ and $w$ is a mapping of $D$ into $Z_{+}$satisfying the following conditions.

(1) For every $i \in I,\{i\} \in D$.

(2) If $J, J^{\prime} \in D$, one of the following cases occurs;
(a) $J \subset J^{\prime}$
(b) $J^{\prime} \subset J$
(c) $J \cap J^{\prime}=\emptyset$.

(3) If $J$ is a maximal element of $D$, then $w(J)=1$.

(4) If $J, J^{\prime} \in D$ and if $J \subseteq J^{\prime}$, then $w(J)$ is a multiple of $w\left(J^{\prime}\right)$ and $w(J)>w\left(J^{\prime}\right)$.

(5) If $J_{1}, J_{2}, J \in D$ and if $J_{i} \prec J(i=1,2)$, then $w\left(J_{1}\right)=w\left(J_{2}\right)$. (We write $J \prec J^{\prime}$ if $J \subseteq J^{\prime}$ and if there is no element of $D$ between $J$ and $J^{\prime}$ )

A datum $D$ is a couple of a special datum $D^{\prime}$ and $\left(a_{1}, \cdots, a_{n}\right) \in Z_{+}^{n}$. We identify a special datum $D$ with the datum $(D,(1, \cdots, 1))$.

Definition 1.2. If $\boldsymbol{D}=\left(D, w,\left(a_{1}, \cdots, a_{n}\right)\right)$ is a datum, we put

$$
R_{D}=C\left[X_{J} ; J \in D\right], \quad \text { where } X_{J}=\left(\prod_{i \in J} X_{i}^{a_{i}}\right)^{w(J)} \text {. }
$$

Definition 1.3. If $\boldsymbol{D}=\left(D, w,\left(a_{1}, \cdots, a_{n}\right)\right)$ is a datum, the group $G_{\boldsymbol{D}}$ is the one generated by the following elements;

$$
\begin{aligned}
& \left\{\left(e_{a_{i}} ; i\right) \mid i \in I\right\} \text { and } \\
& \left\{\left(e_{w a_{i}}, e_{w a_{j}}^{-1} ; i, j\right) \mid J_{1}, J_{2}, J \in D, i \in J_{1}, j \in J_{2}, J_{1}, J_{2} \prec J \text { and } w=w\left(J_{1}\right)=w\left(J_{2}\right)\right\} .
\end{aligned}
$$

Notation 1.4. To illustrate a special datum $D$, we define the graph of $D=(D, w)$ as follows;

(i) We represent $J \in D$ by a circle and we write the integer $w(J)$ inside it.

(ii) If $J \prec J^{\prime}$, we join the corresponding circles by a line segment in such a way that the circle corresponding $J^{\prime}$ lies above that of $J$.

ExAmple 1.5. A. If the graph of $D$ is

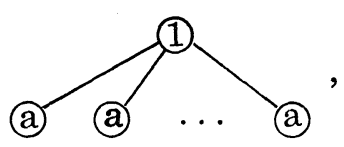


then

$$
\begin{aligned}
& R_{\boldsymbol{D}}=C\left[X_{1}^{a}, \cdots, X_{n}^{a}, X_{1} X_{2} \cdots X_{n}\right] \text { and } \\
& G_{\boldsymbol{D}}=\left\langle\left(e_{a}, e_{a}^{-1} ; 1,2\right),\left(e_{a}, e_{a}^{-1} ; 2,3\right), \cdots,\left(e_{a}, e_{a}^{-1} ; n-1, n\right)\right\rangle .
\end{aligned}
$$

It will be shown later that if $G \subset S L(n, C)$ is a finite Abelian group which is not contained in $S L(n-1, C)$ and if $S^{G}$ is a hypersurface, then $S^{G}=R_{D}$ and $G=G_{D}$ of this example (cf. Theorem 2.1).

B. If the graph of $D$ is

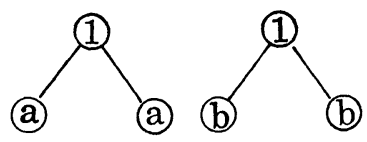

$(n=4)$, then $R_{D}=C\left[X_{1}^{a}, X_{2}^{a}, X_{1} X_{2}, X_{3}^{b}, X_{4}^{b}, X_{3} X_{4}\right]=R_{D_{1}} \otimes_{C} R_{D_{2}}$, where $D_{1}$ and $D_{2}$ are special data whose graphs are

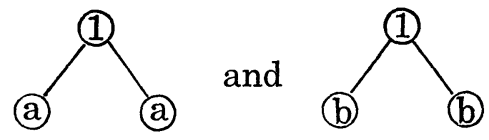

respectively and $G_{\boldsymbol{D}}=\left\langle\left(e_{a}, e_{a}^{-1} ; 1,2\right),\left(e_{b}, e_{b}^{-1} ; 3,4\right)\right\rangle=G_{\boldsymbol{D}_{\mathbf{1}}} \times G_{\boldsymbol{D}_{\mathbf{2}}}$.

Remark 1.6. By the construction, it is clear that if $D$ is a special datum and if $D^{\prime}=\left(D,\left(a_{1}, \cdots, a_{n}\right)\right)$ is a datum, then $R_{D} \cong R_{D^{\prime}}$.

Proposition 1.7. If $\boldsymbol{D}=\left(D, w,\left(a_{1}, \cdots, a_{n}\right)\right)$ is a datum, then

(1) the ring $R_{D}$ is a complete intersection.

(2) $R_{D}$ is the invariant subring under the action of the group $G_{D}$.

Proof. We prove this by induction on the cardinality of $D$. If $\#(D)=n, R_{D}$ is a polynomial ring and the statement (2) is clear, too.

(1) Let $J$ be a maximal element of $D$ with $\#(J) \geqq 2$. We can write $J=J_{1} \cup \cdots \cup J_{p}$, where $J_{i} \prec J$ for $i=1, \cdots, p$. We put $D^{\prime}=D \backslash\{J\}$ and $D^{\prime}=\left(D^{\prime}, w^{\prime},\left(a_{1}^{\prime}, \cdots, a_{n}^{\prime}\right)\right)$, where

$$
w^{\prime}\left(J^{\prime}\right)= \begin{cases}w\left(J^{\prime}\right) / w\left(J_{i}\right) & \left(\text { if } J^{\prime} \subset J_{i}\right) \\ w\left(J^{\prime}\right) & \left(\text { if } J^{\prime} \varnothing J\right)\end{cases}
$$

and

$$
a_{j}^{\prime}= \begin{cases}a_{j} \cdot w\left(J_{i}\right) & \left(\text { if } j \in J_{i}\right) \\ a_{j} & (\text { if } j \notin J) .\end{cases}
$$

Then it is easy to see that $R_{D}=R_{D^{\prime}}\left[X_{J}\right] \cong R_{D^{\prime}}[Y] /\left(Y^{w\left(J_{i}\right)}-\prod_{i=1}^{p} X_{J_{i}}\right)$. As. 
$R_{D^{\prime}}$ is a complete intersection by the induction hypothesis, so is $R_{D}$.

(2) It is easy to see that every element of $R_{D}$ is invariant under the action of $G_{D}$. Also, by easy computation, we can reduce to the case where $D$ is a special datum. As $S^{G}$ is generated by monomials, it suffices to show that every monomial in $S^{G_{D}}$ is divisible by some $X_{J}(J \in D)$. Let $M=X^{c} \quad\left(c=\left(c_{1}, \cdots, c_{n}\right)\right)$ be a monomial in $S^{G}$. We put $I^{\prime}=$ $\left\{i \in I \mid c_{i}>0\right\}$. If $I^{\prime}$ contains some maximal element $J$ of $D$, then $M$ is divisible by $X_{J}$. Otherwise, there exist $J, J^{\prime} \in D$ such that $J^{\prime} \prec J, J^{\prime} \subset I^{\prime}$ and $J \varnothing I^{\prime}$. If we take $j \in J$ so that $j \notin I^{\prime}, s_{i}=\left(e_{w\left(J^{\prime}\right)}, e_{w\left(J^{\prime}\right)}^{-1} ; i, j\right) \in G_{D}$ for every $i \in J^{\prime}$. As $s_{i}(M)=M$, we have $w\left(J^{\prime}\right) \mid c_{i}$ for every $i \in J^{\prime}$. This means that $X_{J^{\prime}}$ divides $M$. (The author thanks the referee for advising him this nice proof.)

Remark 1.8. We can define the ring $R_{D}(k)$ for any field $k$ and a datum $D$ by putting $R_{D}(k)=k\left[X_{J} ; J \in D\right]$. (The definition of $X_{J}$ is the same as the one in 1.2.) The proof of $1.7(1)$ shows that $R_{D}(k)$ is a complete intersection for an arbitrary field $k$.

\section{The main theorem}

In this section, we conserve the notation and conventions at the beginning of Section 1.

THEOREM 2.1. If $G$ is a finite Abelian subgroup of $G L(n, C)$ (resp. $S L(n, C)$ ) and if $S^{G}$ is a complete intersection, then there is a datum (resp. a special datum) $D$ such that $S^{G}=R_{D}$ and $G=G_{D}$.

We divide the proof of (2.1) in several steps.

2.2. As $G$ is diagonal, $S^{G}$ is generated by monomials of $X_{1}, \cdots, X_{n}$. For a monomial $M=X^{a}\left(a=\left(a_{1}, \cdots, a_{n}\right)\right)$, we put $\operatorname{deg}(M)=\left(a_{1}, \cdots, a_{n}\right)$. In this manner, $S^{G}$ is a $Z^{n}$-graded ring. We put $\mathfrak{m}=\left(X_{1}, \cdots, X_{n}\right) S \cap S^{G}$ and we choose monomials $M_{1}, \cdots, M_{n+t}$ so that the images of $M_{i}$ 's in $\mathrm{m} / \mathrm{m}^{2}$ form a basis of $\mathrm{m} / \mathrm{m}^{2}$. It is clear that $M_{i}$ 's are uniquely determined by this condition and that $M_{1}, \cdots, M_{n+t}$ are the minimal generators of $S^{G}$. Among $M_{i}$ 's, there are monomials of the form $X_{i}^{a_{i}}(i=1, \cdots, n)$. So we can assume that $M_{i}=X_{i}^{a_{i}}$ for $i=1, \cdots, n$. As $S^{G}$ is normal, $M_{i}(i=1$, $\cdots, n$ ) are uniquely determined by this property.

Now, let us define the homomorphism

$$
T: C\left[Y_{1}, \cdots, Y_{n+t}\right] \rightarrow S^{G}
$$


by $T\left(Y_{i}\right)=M_{i}(i=1, \cdots, n+t)$. We consider $C\left[Y_{1}, \cdots, Y_{n+t}\right]$ a $Z^{n}$-graded ring by putting $\operatorname{deg}\left(Y_{i}\right)=\operatorname{deg}\left(M_{i}\right)(i=1, \cdots, n+t)$. Then $T$ is a homomorphism of $Z^{n}$-graded rings and so $\operatorname{Ker}(T)$ is a $Z^{n}$-graded ideal. It is easy to see that $\operatorname{Ker}(T)$ is generated by the differences

$$
Y_{1}^{c_{1}} \cdots Y_{n+t}^{c_{n+t}}-Y_{1}^{d_{1}} \cdots Y_{n+t}^{d_{n+t}} \quad \text { such that } M_{1}^{c_{1}} \cdots M_{n+t}^{c_{n+t}}=M_{1}^{d_{1}} \cdots M_{n+t}^{d_{n+t}} .
$$

Minimal basis of $\operatorname{Ker}(T)$ is given by the basis of the vector space $\operatorname{Ker}(T) /\left(Y_{1}, \cdots, Y_{n+t}\right) \operatorname{Ker}(T)$. For $i=n+1, \cdots, n+t$, some power of $M_{i}$ is a product of other $M_{j}^{\prime}$ 's. So, there is a difference

$$
F_{i}=Y_{i}^{b_{i}}-\left(\text { monomial of } Y_{j}^{\prime} \mathrm{s}\right) \quad(i=n+1, \cdots, n+t)
$$

such that $F_{i}$ is a member of a minimal generating set of $\operatorname{Ker}(T)$. As $S^{G}$ is normal, a relation of the type $M_{i}^{p}-M_{j}^{q}=0$ does not occur for $i \neq j$. So $F_{n+1}, \cdots, F_{n+t}$ are distinct elements of $\operatorname{Ker}(T)$. Also, the images of $F_{n+1}, \cdots, F_{n+t}$ in $\operatorname{Ker}(T) /\left(Y_{1}, \cdots, Y_{n+t}\right) \operatorname{Ker}(T)$ are linearly independent since $\operatorname{Ker}(T) /\left(Y_{1}, \cdots, Y_{n+t}\right) \operatorname{Ker}(T)$ is a $Z^{n}$-graded module and $\operatorname{deg}\left(F_{i}\right)$ $(i=n+1, \cdots, n+t)$ are all distinct. If $S^{G}$ is a complete intersection, $\operatorname{Ker}(T)$ is generated by precisely $t$ elements and the above argument shows that $\operatorname{Ker}(T)$ is generated by $F_{n+1}, \cdots, F_{n+t}$.

Before proceeding further, we need some remarks.

2.3. In general, if $R$ is a Gorenstein ring graded by $N^{n}$ and if $R_{0}$ is a field, then the canonical module $K_{R}$ of $R$ has the natural $Z^{n}$-graded $R$-module structure and $K_{R}=R(d)$ for some $d=\left(d_{1}, \cdots, d_{n}\right) \in Z^{n}$ as $Z^{n}$ graded $R$-modules (cf. [3]). We define $a(R)=d$. This invariant $a(R)$ plays an essential role in the proof of 2.1. We need two facts concerning $a(R)$.

2.4. (R. Stanley, [5]) $K_{\left(S^{G}\right)}=\left(S^{G}\right)_{+}$as $Z^{n}$-graded $S^{G}$-modules, where $\left(S^{G}\right)_{+}$is the ideal generated by $\left\{X^{e} \in S^{G} \mid e=\left(e_{1}, \cdots, e_{n}\right), e_{i}>0(i=1, \cdots, n)\right\}$.

ExAmples. A. If $G \subset S L(n, C)$, then $S^{G}$ is a Gorenstein ring and $a\left(S^{G}\right)=(-1, \cdots,-1)$. Conversely, if $S^{G}$ is a Gorenstein ring and $a\left(S^{G}\right)$ $=(-1, \cdots,-1)$, then $G \subset S L(n, C)$.

B. If $\boldsymbol{D}=\left(D, w,\left(a_{1}, \cdots, a_{n}\right)\right)$ is a datum, then $a\left(R_{\boldsymbol{D}}\right)=\left(-a_{1}, \cdots,-a_{n}\right)$.

2.5. If $R=k\left[Y_{1}, \cdots, Y_{n+t}\right] /\left(F_{1}, \cdots, F_{t}\right)$ is a complete intersection, where $k$ is a field and $\operatorname{deg}\left(Y_{i}\right) \in N^{n} \backslash\{0\}$ is given so that $F_{1}, \cdots, F_{t}$ are homogeneous with respect to this grading, then 


$$
a(R)=\sum_{i=1}^{t} \operatorname{deg}\left(F_{i}\right)-\sum_{j=1}^{n+t} \operatorname{deg}\left(Y_{j}\right) .
$$

The proof of this fact is the same as those of (2.2.8) and (2.2.10) in [2].

2.6. If $J$ is a subset of $I$ and if we put $S_{J}=C\left[X_{i} ; i \in J\right]$, then $G$. acts on $S_{J}$ (as we have assumed that $G$ is diagonal) and $\left(S_{J}\right)^{G}=S_{J} \cap S^{G}$. And,

2.7. If $S^{G}$ is a complete intersection, then $\left(S_{J}\right)^{G}$ is a complete intersection for every subset $J$ of $I$.

Proof. In the notation of 2.2, $\left(S_{J}\right)^{G}=C\left[M_{i} \mid M_{i} \in S_{J}\right]$. If we define

$$
T_{J}: C\left[Y_{i} \mid 1 \leqq i \leqq n+t, M_{i} \in S_{J}\right] \rightarrow\left(S_{J}\right)^{a}
$$

by $T_{J}\left(Y_{i}\right)=M_{i}$, then it is easy to show that the set $\left\{F_{i} \mid n+1 \leqq i \leqq n+t\right.$, $\left.M_{i} \in S_{J}\right\}$ generate $\operatorname{Ker}\left(T_{J}\right)$.

2.8. Let $G$ be a (not necessarily Abelian) finite subgroup of $G L(n, C)$. The following facts are known.

THEOREM [1]. $S^{G}$ is a polynomial ring if and only if $G$ is generated by its pseudo-reflections. ( $g \in G$ is a pseudo-reflection if $\operatorname{rank}(g-I)=1$.)

TheOREM [7]. If $G$ contains no pseudo-reflections, then $S^{G}$ is a Gorenstein ring if and only if $G \subset S L(n, C)$.

If $G$ is Abelian (and diagonal); then every pseudo-reflection in $G$ is of the form $(e ; i)$ where $e$ is a root of unity and $i \in I$. If $H$ is the subgroup of $G$ generated by all the pseudo-reflections of $G$, then

$$
S^{H}=C\left[X_{1}^{a_{1}}, \cdots, X_{n}^{a_{n}}\right]
$$

for some integers $a_{1}, \cdots, a_{n}$. The group $G / H$ acts linearly on the new basis $\left(X_{1}^{a_{1}}, \cdots, X_{n}^{a_{n}}\right)$. If $S^{G}$ is a Gorenstein ring, then $G / H \subset S L(n, C)$ by this new representation. That is, $X_{1}^{a_{1}} \cdots X_{n}^{a_{n}} \in S^{a}$. So, to prove 2.1, we may assume that $G \subset S L(n, C)$.

2.9. Now, let us continue the proof of Theorem 2.1. We assume that $G \subset S L(n, C)$ and that $S^{G}$ is a complete intersection. We put $S^{G}=$ $C\left[M_{1}, \cdots, M_{n+t}\right]$ as in 2.2. We prove the theorem by induction on $n$. (For $n \leqq 2$, the conclusion of 2.1 is well known and is easy to prove.) So, we assume that for $J \subseteq I,\left(S_{J}\right)^{G}=R_{D}$ for a datum $D$ for the index 
set $J\left(\left(S_{J}\right)^{G}\right.$ is a complete intersection by 2.7$)$.

For a monomial $M$, we define $\operatorname{Supp}(M)=\left\{i \in I\left|X_{i}\right| M\right\}$. We put $\operatorname{Supp}\left(M_{i}\right)=J_{i}(i=1, \cdots, n+t)$.

2.10. If $i \neq j$, then $J_{i} \neq J_{j}$.

Proof. Assume that $J=J_{i}=J_{j}$. Considering the action of $G$ on $S_{J}$ and by the induction hypothesis, we may assume $J=I$. But in this case, as $X_{1} \cdots X_{n} \in S^{G}$, the only possible monomial $M$ with $\operatorname{Supp}(M)=I$ that is a member of the minimal generators of $S^{G}$ is $M=X_{1} \cdots X_{n}$. A contradiction!

2.11. If $i \neq j$, either $J_{i} \subset J_{j}, J_{i} \supset J_{j}$ or $J_{i} \cap J_{j}=\emptyset$.

Proof. If the conclusion is false, there is a pair $(i, j)$ such that $J_{i} \not \supset J_{j}, \quad J_{i} \varangle J_{j}$ and $J=J_{i} \cap J_{j} \neq \emptyset$. Let us take such a pair that $J_{i} \cup J_{j}$ is minimal. By the induction hypothesis, we may assume $J_{i} \cup J_{j}$ $=I$. Then $P=X_{1} \cdots X_{n}$ divides $M_{i} M_{j}$. So, by $2.2, P$ must be a member of the minimal generating set of $S^{G}$ and there is an integer $a$ such that $P^{a}=M_{i} M_{j}$. Also by 2.2 , there is no further couple $(k, m)$ such that $J_{k} \cup J_{m}=I$. By the minimality assumption, for every $k(k=1, \cdots, n+t)$, one of the following cases occurs; (i) $M_{k}=P$ (ii) $J_{k} \subset J_{i}$ (iii) $J_{k} \subset J_{j}$. That is, we have $S^{G}=C\left[\left(S_{J_{i}}\right)^{G},\left(S_{J_{j}}\right)^{G}, P\right]$. By the induction hypothesis, there exist data $D_{i}$ and $D_{j}$ for the index sets $J_{i}$ and $J_{j}$, respectively, such that $\left(S_{J_{i}}\right)^{G}=R_{D_{i}}$ and $\left(S_{J_{j}}\right)^{G}=R_{D_{j}}$. We want to compute $a\left(S^{G}\right)$ to have a contradiction. Consider the homomorphism $T$ defined in 2.2. We have seen in 2.2 that $\operatorname{Ker}(T)=\left(F_{n+1}, \cdots, F_{n+t}\right)$. If $k \geqq n+1$ and if $J_{k} \subset J_{i}$, then, by the construction of $R_{D_{i}}, F_{k}=Y_{k}^{v}-\prod_{m \in J_{k}^{\prime}} Y_{m}$, where $J_{k}^{\prime}=\left\{m \mid J_{m}\right.$ $\left.\prec J_{k}\right\}$ and $v=w\left(J_{m}\right) / w\left(J_{k}\right)$ (everything is considered in the datum $\boldsymbol{D}_{i}$ ). The situation is similar if $J_{k} \subset J_{j}$. Thus we have

$$
\operatorname{deg}\left(F_{k}\right)=\sum_{J_{m}<J_{k}} \operatorname{deg}\left(M_{m}\right) .
$$

If $M_{k}=P$, we have seen $F_{k}=P^{a}-M_{i} M_{j}$. By 2.5,

$$
a\left(S^{G}\right)=\sum_{k=n+1}^{n+t} \operatorname{deg}\left(F_{k}\right)-\sum_{m=1}^{n+t} \operatorname{deg}\left(M_{m}\right) .
$$

We recall that $\left\{J_{k} \mid k=1, \cdots, n+t\right\}=D_{i} \cup D_{j} \cup\{I\}$ and that $D_{i}$ and $D_{j}$ have the non-empty intersection. If we replace $\operatorname{deg}\left(F_{k}\right)$ by the equality $\left(^{*}\right)$, $\operatorname{deg}\left(M_{m}\right)$ appears twice if $J_{m} \prec J_{k}$ in $D_{i}$ and $J_{m} \prec J_{k^{\prime}}$ in $D_{j}$ for some $J_{k}$ and $J_{k^{\prime}}$. So, we have 
$a\left(S^{a}\right)=-\operatorname{deg}(P)+\sum\left\{\operatorname{deg}\left(M_{k}\right) \mid J_{k}\right.$ is a maximal element of $\left.J=J_{i} \cap J_{j}\right\}$.

But, on the other hand, as $G \subset S L(n, C)$, we must have $a\left(S^{G}\right)=-\operatorname{deg}(P)$ $=(-1, \cdots,-1)$. This contradicts the fact that $J \neq \emptyset$.

2.12. For every $i, i=1, \cdots, n+t, M_{i}=\left(\prod_{m \in J i} X_{m}\right)^{w_{i}}$ for some integer $w_{i}$ and $w_{i}=w_{j}$ if $J_{i} \prec J_{k}$ and $J_{j} \prec J_{k}$ for some $J_{k}$.

Proof. We prove this by descending induction on $J_{i}$. If $J_{i}=I$, then $M_{i}=P$ and we have already seen that $P^{a}=\prod_{J_{k}<I} M_{k}$ for some integer $a$ (cf. 2.11). Thus $M_{k}=\left(\prod_{i \in J_{k}} X_{i}\right)^{a}$ for every $k$ such that $J_{k} \prec I$. We can repeat this process considering the action of $G$ on $S_{J_{i}}$ and using the induction hypothesis. Also, this argument shows that we have checked the condition (4) of 1.1. The proof of Theorem 2.1 is complete.

Example 2.13. To illustrate the proof of 2.11, let us give an example. If we put $R=C\left[X^{4}, Y^{2}, Z^{4}, X^{2} Y, Y Z^{2}, X Y Z\right], R$ is a complete intersection and $a(R)=(-1,1,-1)=(-1,-1,-1)+(0,2,0)$. The calculation of $a(R)$ shows that $R$ is not normal. The normalization of $R$ is $R\left[X^{3} Z, X^{2} Z^{2}, X Z^{3}\right]$, which is not a complete intersection.

Remark 2.14. Let $H \subset N^{n}$ be a finitely generated additive semigroup and let $k$ be a field. Then the property " $R=k[H]\left(k[H]=k\left[X^{h} ; h=\right.\right.$ $\left.\left.\left(h_{1}, \cdots, h_{n}\right) \in H\right]\right)$ is a complete intersection" does not depend on $k$. So, we have the following

THEOREM. Let $k$ be a field and $H=N^{n} \cap L$ be a semigroup, where $L$ is an additive subgroup of $Z^{n}$ with $\operatorname{rank}(L)=n$. If $R=k[H]$ is $a$ complete intersection, then $R=R_{D}(k)$ for some datum $D$ (cf. 1.8).

Remark 2.15. For $n=3$, normal semigroup rings of dimension 3 over arbitrary field, which are complete intersections were classified by M.-N. Ishida in [4].

Remark 2.16. R. Stanley gave a criterion for $S^{G}$ to be a complete intersection in [6], where $G$ is the intersection of a reflection group $\bar{G}$ and $S L(n, C)$. If $\bar{G}$ is Abelian, $\bar{G}$ is necessarily of the form $\bar{G}_{B}=\left\langle\left(e_{b_{i}} ; i\right)\right|$ $1 \geqq i \geqq n\rangle$, where $B=\left(b_{1}, \cdots, b_{n}\right)$ is an $n$-tuple positive integers. In this case, his criterion says that $S^{G_{B}}\left(G_{B}=\bar{G}_{B} \cap S L(n, C)\right)$ is a complete intersection if and only if the set $\left\{b_{1}, \cdots, b_{n}\right\}$ is "completely reducible" (see [6] for the definition of this word). By our Theorem 2.1, it is not 
hard to get the special case of his theorem when $\bar{G}$ is Abelian. But, for a special datum $D$, the group $G_{D}$ is not necessarily the intersection of a reflection group and $S L(n, C)$.

\section{Some concluding remarks and conjectures}

Proposition 3.1. If $G \subset S L(n, C)$ is a finite Abelian group and if $S^{G}$ is a complete intersection, then

(1) $G$ is generated by $\{g \in G \mid \operatorname{rank}(g-I)=2\}$,

(2) $S^{G}$ is generated by at most $2 n-1$ elements,

(3) if $S^{G}$ is a hypersurface (if $S^{G}$ is generated by $(n+1)$-elements), then $G=\bar{G} \cap S L(n, C)$, where $\bar{G}$ is a finite Abelian reflection group,

(4) if $S^{G}$ is a hypersurface, the multiplicity of $S^{G}$ is at most $n$. If $S^{G}$ is generated by exactly $2 n-1$ elements (if the embedding dimension of $S^{G}$ is $\left.2 n-1\right)$, the multiplicity of $S^{G}$ is $2^{n-1}$. In general, the multiplicity of $S^{G}$ is at most $2^{n-1}$.

Proof. We may assume that $S^{G}=R_{D}$, where $D=(D, w)$ is a special datum. Then (1) is clear by the definition of $G_{D}$. To prove (2)-(4), we may assume that $S^{G}$ does not contain any non-zero linear forms. For $J \in D,|J| \geqq 2$, we put

$$
\delta(J)=\#\left\{J^{\prime} \in D \mid J^{\prime} \prec J\right\} \quad \text { and } \quad m(D)=\prod_{\substack{J \in D \\|J| \geq 2}} \delta(J) .
$$

Then we have $\sum_{J \in D,|J| \geqq 2}(\delta(J)-1)=n-1$. This proves (2). As for (3), if $R_{\boldsymbol{D}}$ is a hypersurface, then $G_{\boldsymbol{D}}=\left\langle\left(e, e_{m}^{-1} ; i, i+1\right) \mid i=1, \cdots, n-1\right\rangle=$ $\left\langle\left(e_{m} ; i\right) \mid i=1, \cdots, n\right\rangle \cap S L(n, C)$ for some integer $m$. To prove (4), we consider the ring $A=R_{D} / \mathfrak{a}$, where $\mathfrak{a}$ is the ideal of $R_{D}$ generated by

$$
\begin{aligned}
& \left\{X_{J} \mid J \text { is a maximal element of } D\right\} \text { and } \\
& \left\{X_{J^{\prime}}-X_{J^{\prime \prime}} \mid J^{\prime}, J^{\prime \prime} \in D \text { and } J^{\prime} \prec J, J^{\prime \prime} \prec J \text { for some } J \in D\right\} .
\end{aligned}
$$

Then $A$ is an Artinian ring and length $(A)=m(D)$ by using the following easy lemma repeatedly, and so the multiplicity of $R_{D}$ is at most $m(D)$. It is easy to see that $m(D) \leqq 2^{n-1}$.

Lemma. If $B$ is an Artinian ring and if $C=B[Y] /\left(Y^{m}-b\right)$, where $Y$ is an indeterminate and $b \in B$, then length $(C)=m$. length $(B)$.

In general, we have the following

Conjecture 3.2. If $G \subset S L(n, C)$ is a (not necessarily Abelian) finite 
group and if $S^{G}$ is a complete intersection, then the followings are true.

(1) $G$ is generated by $\{g \in G \mid \operatorname{rank}(g-I)=2\}$.

(2) The embedding dimension of $S^{G}$ is at most $2 n-1$.

(3) If $S^{G}$ is a hypersurface, then $G=\bar{G} \cap S L(n, C)$ for some finite reflection group $\bar{G}$.

(4) The multiplicity of $S^{G}$ is at most $2^{n-1}$. If $S^{G}$ is a hypersurface, then the multiplicity of $S^{G}$ is at most $n$.

We have examined this conjecture when $G$ is Abelian. If $n=2$, it is well known that $S^{G}$ is a hypersurface of multiplicity 2 for every finite subgroup $G$ of $S L(n, C)$. In [8], we will show that the conjecture is true for $n=3$.

\section{REFERENCES}

[1] C. Chevalley, Invariants of finite groups generated by reflections, Amer. J. Math. 67 (1955), 778-782.

[2] S. Goto and K. Watanabe, On graded rings, I. J. Math. Soc. Japan 30 (1978), 179-213.

[ 3 ] - On graded rings, II. ( $Z^{n}$-graded rings.) Tokyo J. Math. 1 (1978), 237-261.

[4] M.-N. Ishida, Torus embeddings and dualizing complexes, to appear.

[ 5 ] R. Stanley, Hilbert functions of graded algebras. Adv. in Math. 28 (1978), 57-83.

[6] - Relative invariants of finite groups generated by pseudoreflections, J. Alg. 49 (1977), 134-148.

[7] K. Watanabe, Certain invariant subrings are Gorenstein, I, II, Osaka J. Math. 11 (1974), 1-8, 379-388.

[ 8 ] - Invariant subrings of $C[X, Y, Z]$ which are complete intersections, (in preparation).

Tokyo Metropolitan University 